Research Paper

\title{
Retrospective Analysis of the Relationship between Decline in FEV, and Abdominal Circumference in Male Smokers: the Takahata Study
}

\author{
Masamichi Sato ${ }^{1}$, Yoko Shibata ${ }^{1 凶}$, Shuichi Abe ${ }^{1}$, Sumito Inoue ${ }^{1}$, Akira Igarashi $^{1}$, Keiko Yamauchi ${ }^{1}$, Yasuko

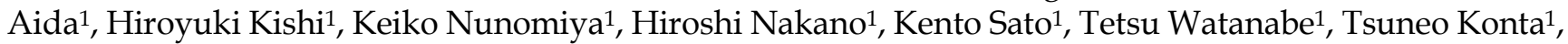 \\ Yoshiyuki Ueno르, Takeo Kato², Takamasa Kayama², Isao Kubota ${ }^{1}$ \\ 1. Department of Cardiology, Pulmonology, and Nephrology; \\ 2. Global Center of Excellence Program Study Group, Yamagata University School of Medicine, 2-2-2 Iida-Nishi Yamagata 990-9585, Japan. \\ $\square$ Corresponding author: Dr. Yoko Shibata, 2-2-2 Iida-Nishi, Yamagata City, Yamagata 990-9585, Japan. Telephone: +81-23-628-5302, FAX: \\ +81-23-628-5305, Email: shibata@med.id.yamagata-u.ac.jp. \\ ( ) Ivyspring International Publisher. This is an open-access article distributed under the terms of the Creative Commons License (http://creativecommons.org/ \\ licenses/by-nc-nd/3.0/). Reproduction is permitted for personal, noncommercial use, provided that the article is in whole, unmodified, and properly cited.
}

Received: 2012.08.09; Accepted: 2012.10.31; Published: 2012.12.05

\begin{abstract}
Background: Metabolic syndrome (Mets) is reportedly associated with chronic obstructive pulmonary disease (COPD). However, the relationship between abdominal circumference (AC) and decline in FEV, has not been elucidated. We aimed to investigate this relationship among male current smokers.

Methods: Spirometry was performed on subjects $(n=3,257) \geq 40$ years of age, who participated in a community-based annual health check in Takahata, Japan, from 2004 through 2006 (visit I). Spirometry was re-evaluated, and AC was assessed in I47 of the male current smokers in 2009 (visit 2). The diagnosis of Mets was based on the criteria used in the Hisayama Study.

Results: No significant relationships were observed between $A C$ and spirometric parameters such as \% predicted forced vital capacity (FVC), \% predicted forced expiratory volume in I s $\left(\mathrm{FEV}_{1}\right)$ and $\mathrm{FEV} / \mathrm{FVC}$. However, decline in FEV , was significantly correlated with AC. Multivariate logistic regression analysis showed that $A C$ was a significant discriminating factor for decline in $\mathrm{FEV}_{1}$, independently of age, Brinkman index and change in body mass index from

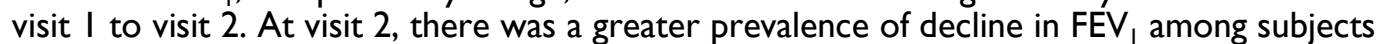
with Mets $(n=17)$ than among those without Mets. Although there were no differences in \% predicted FVC, \% predicted FEV, or FEV/FVC between subjects with or without Mets, the rate of decline in FEV, was significantly greater in subjects with Mets than in those without.

Conclusions: This retrospective analysis suggested that measuring AC may be useful for discriminating male smokers who show a decline in $\mathrm{FEV}_{1}$.
\end{abstract}

Key words: decline in $\mathrm{FEV}_{1}$, abdominal circumference, smoker, health check.

\section{Introduction}

Long-term cigarette smoking is most important cause of chronic obstructive pulmonary disease (COPD) (1). In patients with COPD, forced expiratory volume in $1 \mathrm{~s}\left(\mathrm{FEV}_{1}\right)$ is progressively reduced as the disease develops. In addition, patients with COPD have various systemic comorbidities, including atherosclerosis, cachexia, diabetes mellitus, and osteoporosis (2-7). These comorbidities are thought to be associated with systemic inflammation due to leakage of mediators from sites of local inflammation in the 
lung, and this is an important determinant of the morbidity associated with the disease (8). Recently, metabolic syndrome (Mets) has received attention as a comorbidity of COPD (9-12).

Mets is also thought to be a disease of systemic inflammation that is derived from visceral fat (13). Patients with Mets have higher risks of cardiovascular disease, stroke, and death, compared to subjects without Mets (14). Since Mets and COPD both involve states of systemic inflammation, it is conceivable that these diseases share common inflammatory pathways.

The results of recent cross-sectional investigations from China and France suggested that the central obesity component of Mets was associated with impairment of pulmonary function $(10,15)$. However, it was not clear whether Mets is a risk factor for decline in pulmonary function parameters such as $\mathrm{FEV}_{1}$, among healthy smokers. We hypothesized that excess abdominal circumference (AC), one of the major signs of Mets, is associated with a decline in $\mathrm{FEV}_{1}$ among smokers. To examine this hypothesis, we retrospectively investigated the relationship between $\mathrm{AC}$ and the rate of decline in $\mathrm{FEV}_{1}$.

\section{Methods}

\section{Study population}

This study formed part of the Molecular Epidemiological Study of the Regional Characteristics of $21^{\text {st }}$ Century Centers of Excellence (COE) Program and the Global Centers of Excellence Program in Japan (16). The study was approved by the institutional ethics committee and all participants gave written informed consent.

The study was based on an annual community health check, in which all residents of Takahata town in northern Japan, who were aged 40 years or older, were invited to participate. We have previously reported cross-sectional analyses of spirometry data for subjects who were included in the database from 2004 through 2005 ( $\mathrm{n}=3,165$ : 1,380 males; 1735 females) (16-20). We continued to enroll subjects from Takahata in 2006, and recently the additional 2006 database became available for analysis. From 2004 through 2006 (visit 1), 1,579 males and 1,941 females (a total of 3,520 subjects) were enrolled in the study, and performed initial spirometry. Two-hundred and sixty-three subjects were excluded from the analysis because their spirometry data did not meet the specified criteria. The data for a total of 3,257 subjects $(1,502$ males, 1,755 females) was entered into the final statistical analysis. Subjects used a self-report questionnaire to document their medical histories, smoking habits, current use of medications, and clinical symptoms. The lifetime consumption of cigarettes was expressed as the Brinkman index (number of cigarettes per day $x$ years of smoking). One-hundred and forty-seven of the 523 male current smokers (visit 1) performed subsequent follow-up spirometry in 2009 (visit 2). Body mass index (BMI), Brinkman index, mean blood pressure (BP), triglyceride (TG), high-density lipoprotein-cholesterol (HDL-c), fasting blood sugar (FBS), \% predicted forced vital capacity [FVC (\%predicted)], and \% predicted forced expiratory volume in $1 \mathrm{~s}\left[\mathrm{FEV}_{1}\right.$ (\% predicted)] were not significantly different between these 147 male current smokers and the remaining 376 male smoker who did not participate in follow-up spirometry in 2009 (data not shown). However, the mean age of these 147 participants was significantly lower than that of the 376 non-participants assessed in 2004-2006 [mean age (years): $56.40 \pm 9.06$ vs. $61.09 \pm 10.59, \mathrm{P}<0.0001]$.

\section{Measurements}

AC around the navel was determined at visit 2 using a tape measure. Systolic and diastolic BP were measured using a mercury sphygmomanometer, which was placed on the right arm of seated subjects, who had rested in a sitting position for at least $5 \mathrm{~min}$ before the measurement. Measurements were performed twice. Blood samples were taken from the antecubital vein of subjects who had been fasting, and the samples were immediately transferred to chilled tubes.

The diagnosis of Mets was based on the criteria used in the Hisayama Study, a Japanese cohort study in a general population: abdominal obesity with abdominal circumference $>90 \mathrm{~cm}$ in men; elevated blood pressure with average systolic/diastolic blood pressures $\geq 130 / 85 \mathrm{~mm} \mathrm{Hg}$; hypertriglyceridemia, defined as a serum TG level $\geq 150 \mathrm{mg} / \mathrm{dL}$; a low HDL-c level, defined as $<40 \mathrm{mg} / \mathrm{dL}$ in men; elevated blood glucose with a FBS level $\geq 110 \mathrm{mg} / \mathrm{dL}$. Metabolic syndrome was defined as the presence of three or more of these factors (21).

Spirometric parameters (FVC and $\mathrm{FEV}_{1}$ ) were measured using standard techniques, with subjects performing FVC maneuvers on a CHESTAC-25 part II EX instrument (Chest Corp., Tokyo, Japan), according to the guidelines of the Japanese Respiratory Society (JRS) (22). Bronchodilator was not administered prior to spirometry. The highest value from at least three FVC maneuvers by each subject was used for the analysis. The results were assessed by two pulmonary physicians, who visually inspected the flow-volume curves and excluded subjects with inadequate data, as defined by the JRS criteria (22). The rate of decline in 
spirometric measures $\left[\Delta \mathrm{FEV}_{1}(\%\right.$ predicted $) /$ year (\%/year) and $\triangle \mathrm{FVC}$ (\% predicted)/year (\%/year)] were calculated as $[(\%$ predicted value at visit 2 - \% predicted value at visit 1$) / \%$ predicted value at visit 1] $\times 100 /$ time between observations (years).

\section{Statistical analyses}

The student's t-test for parametric data or the Mann-Whitney $U$ test for non-parametric data were used to analyze the differences between two groups of subjects. The chi-square test was used to evaluate differences in proportions. All statistical analyses, including linear regression and logistic regression analyses were performed using JMP version 8 software (SAS Institute Inc., Cary, NC, USA). Data are presented in the Figures and Tables as means (SD) or medians (interquartile range). Significance was inferred for differences with $P$ values $<0.05$.

\section{Results}

\section{Characteristics of the subjects}

The characteristics of the male smoking subjects who participated in this study and performed spirometry at both visit 1 and visit 2 are summarized in Table 1. Mean BMI did not differ between visit 1 and visit 2. Mean FVC (\% predicted) at visit 2 was significantly higher than that at visit 1 , although $\mathrm{FEV}_{1}(\%$ predicted) at visit 2 did not differ from that at visit 1 . $\mathrm{FEV}_{1} / \mathrm{FVC}$ was significantly lower at visit 2 than at visit 1 , and the prevalence of airflow limitation was significantly greater at visit 2 than at visit 1 (Table 1 ). There was a strong correlation between $\mathrm{BMI}$ and $\mathrm{AC}$ at visit $2(r=0.89, P<0.0001)$.

Table I. Characteristics of the study subjects at visit I and visit 2.

\begin{tabular}{lll}
\hline & Visit 1 & Visit 2 \\
\hline Age, years & $56.4(9.1)$ & $60.9(9.1)^{\star * *}$ \\
BMI, kg/m ${ }^{2}$ & $22.8(2.8)$ & $22.9(2.9)$ \\
AC, cm & Not measured & $83.5(8.7)$ \\
Brinkman index, cigarette years & $751.7(413.8)$ & $847.9(449.5)^{\star * *}$ \\
FVC, \% predicted & $94.4(13.5)$ & $98.5(14.4)^{\star *}$ \\
FEV $_{1}, \%$ predicted & $92.4(15.8)$ & $91.9(17.1)$ \\
FVEV $_{1} /$ FVC, \% & $77.3(8.4)$ & $73.0(9.3)^{\star * *}$ \\
FEV $_{1} /$ FVC $<0.7, \mathrm{n}(\%)$ & $23(15.6)$ & $48(32.7)^{\star *}$ \\
\hline
\end{tabular}

A total of 147 subjects were included in the study. AC data were not available for nine subjects. Brinkman index data were not available for 19 subjects due to the lack of precise information about cigarette smoking habit. Values are means (SD) or number (\%) of subjects. Difference in Brinkman index between visit 1 and visit 2 was evaluated by the Mann-Whitney $U$ test. Difference in the proportion of $\mathrm{FEV}_{1} / \mathrm{FVC}<0.7$ was evaluated by the chi-square test. Differences in other variables were evaluated by Student's t-test. ${ }^{* *} P<0.001$, *** $P<0.0001$ compared with data for visit 1 . BMI, body mass index; AC, abdominal circumference; FVC, forced vital capacity; $\mathrm{FEV}_{1}$, forced expiratory volume in $1 \mathrm{~s}$

\section{Relationship between abdominal circumfer- ence and spirometric measures}

The relationship between $\mathrm{AC}$ and spirometric measures, and that between BMI and spirometric measures was investigated. No significant correlations between $\mathrm{AC}$ and unadjusted spirometric measures, and between BMI and unadjusted spirometric measures were detected (data not shown). In addition, there were no significant correlations between $\mathrm{AC}$ and adjusted spirometric measures for Brinkman index and status of Mets (Table 2A), and nor was BMI correlated with adjusted spirometric measures (Table 2B). The relationships between AC and changes in spirometric values were also evaluated. As shown in Figure 1, AC was inversely correlated with changes in $\mathrm{FEV}_{1}$ (\% predicted) $(r=-0.19, P=$ 0.024; Figure 1B), but not with changes in FVC (\% predicted) (Figure 1A). However, there were no correlations between BMI and the changes in FVC (\% predicted) or $\mathrm{FEV}_{1}$ (\% predicted) (Figures 1C \& 1D). In addition, the relationship between changes in spirometric values and change in $\mathrm{BMI}\left(\triangle \mathrm{BMI}=\mathrm{BMI}_{\text {visit2 }}\right.$ $B M I_{\text {visit1) }}$ were investigated, since weight gain may reduce spirometric values (23). Both $\triangle \mathrm{FVC}$ (\% predicted)/year and $\triangle \mathrm{FEV}_{1}$ (\% predicted)/year did not correlated with $\triangle \mathrm{BMI}[\Delta \mathrm{FVC}(\%$ predicted $) /$ year: $r=$ -0.06, $P=0.44 ; \Delta \mathrm{FEV}_{1}$ (\% predicted)/year: $r=-0.11, P$ $=0.16]$. We set the cut-off value for decline in $\mathrm{FEV}_{1}(\%$ predicted) as a reduction from baseline of $\geq 1.6 \%$, because this was the lowest quintile of $\Delta \mathrm{FEV}_{1}$ (\% predicted)/year. Multiple logistic regression analysis revealed that AC significantly discriminated subjects who showed a decline in $\mathrm{FEV}_{1}$, independently of age, cigarette consumption (Brinkman index), and $\triangle \mathrm{BMI}$ (Table 3).

Table 2. Relationship of abdominal circumference and body mass index with spirometric measures at visit 2 .

\begin{tabular}{lll}
\hline & Coefficient & $P$ value \\
\hline A. Abdominal circumference & & \\
FVC, \% predicted & 0.124 & 0.48 \\
FEV $_{1}, \%$ predicted & 0.017 & 0.93 \\
FEV $_{1} /$ FVC & -0.050 & 0.66 \\
B. BMI & & \\
FVC, \% predicted & 0.584 & 0.26 \\
FEV1, \% predicted & 0.609 & 0.31 \\
FEV1/FVC & 0.261 & 0.44 \\
\hline
\end{tabular}

Correlations between spirometric measurements and (A) abdominal circumference or (B) BMI were shown. Results were adjusted for Brinkman index and status of metabolic syndrome using multivariate linear regression assay. $\mathrm{BMI}$, body mass index; FVC, forced vital capacity; $\mathrm{FEV}_{1}$, forced expiratory volume in $1 \mathrm{~s}$ 
Table 3. Multivariate logistic regression analysis for discrimination of subjects showing a decline in FEV .

\begin{tabular}{llll}
\hline & OR & $95 \%$ CI & $P$ value \\
\hline $\begin{array}{l}\text { Age (per 1-year increase) } \\
\text { Brinkman Index (per }\end{array}$ & 1.043 & $(0.976-1.120)$ & 0.217 \\
$\begin{array}{l}\text { 1-cigarette year increase) } \\
\text { AC (per 1-cm increase) }\end{array}$ & 1.000 & $(0.999-1.001)$ & 0.479 \\
$\begin{array}{l}\Delta \text { BMI (per 1-kg } / \mathrm{m}^{2} \text { in- } \\
\text { crease) }\end{array}$ & 1.002 & $(1.027-1.178)$ & 0.005 \\
\hline
\end{tabular}

Out of a total of 147 subjects, abdominal circumference data were not available for nine subjects. Brinkman index data were not available for 19 subjects due to the lack of precise information about cigarette smoking habit. $\triangle \mathrm{BMI}=$ $\mathrm{BMI}_{\text {visit2 }}$ - BMI visit1 $_{1} \mathrm{AC}$, abdominal circumference; BMI, body mass index; $\mathrm{FEV}_{1}$, forced expiratory volume in $1 \mathrm{~s}$

\section{Metabolic syndrome and decline in FEV}

A diagnosis of Mets could not be made for 10 of the 147 subjects due to the lack of necessary infor- mation (AC for 9; FBS level for 1). The differences in subject characteristics according to whether or not they had Mets are shown in Table 4. Subjects with Mets were younger, and had a greater BMI, $\triangle \mathrm{BMI}$, $\mathrm{AC}$, systolic $\mathrm{BP}$, diastolic $\mathrm{BP}$, and higher TG levels (Table 4). HDL-c levels were significantly lower in subjects with Mets than in those without Mets; however FBS levels and Brinkman index did not differ between subjects with or without Mets (Table 4). A decline in $\mathrm{FEV}_{1}$ (\% predicted) was more prevalent among subjects with Mets compared to those without Mets (Table 5). In addition, the median decline in $\mathrm{FEV}_{1}$ (\% predicted) was significantly greater in subjects with Mets than it was in those without Mets (Table 6). Spirometric measures at visit 2 did not differ significantly between subjects with or without Mets (Table 6). Even after adjustment of $\mathrm{FEV}_{1} / \mathrm{FVC}$ for age, there was no difference in $\mathrm{FEV}_{1} / \mathrm{FVC}$ between the two groups $(P=0.91)$.
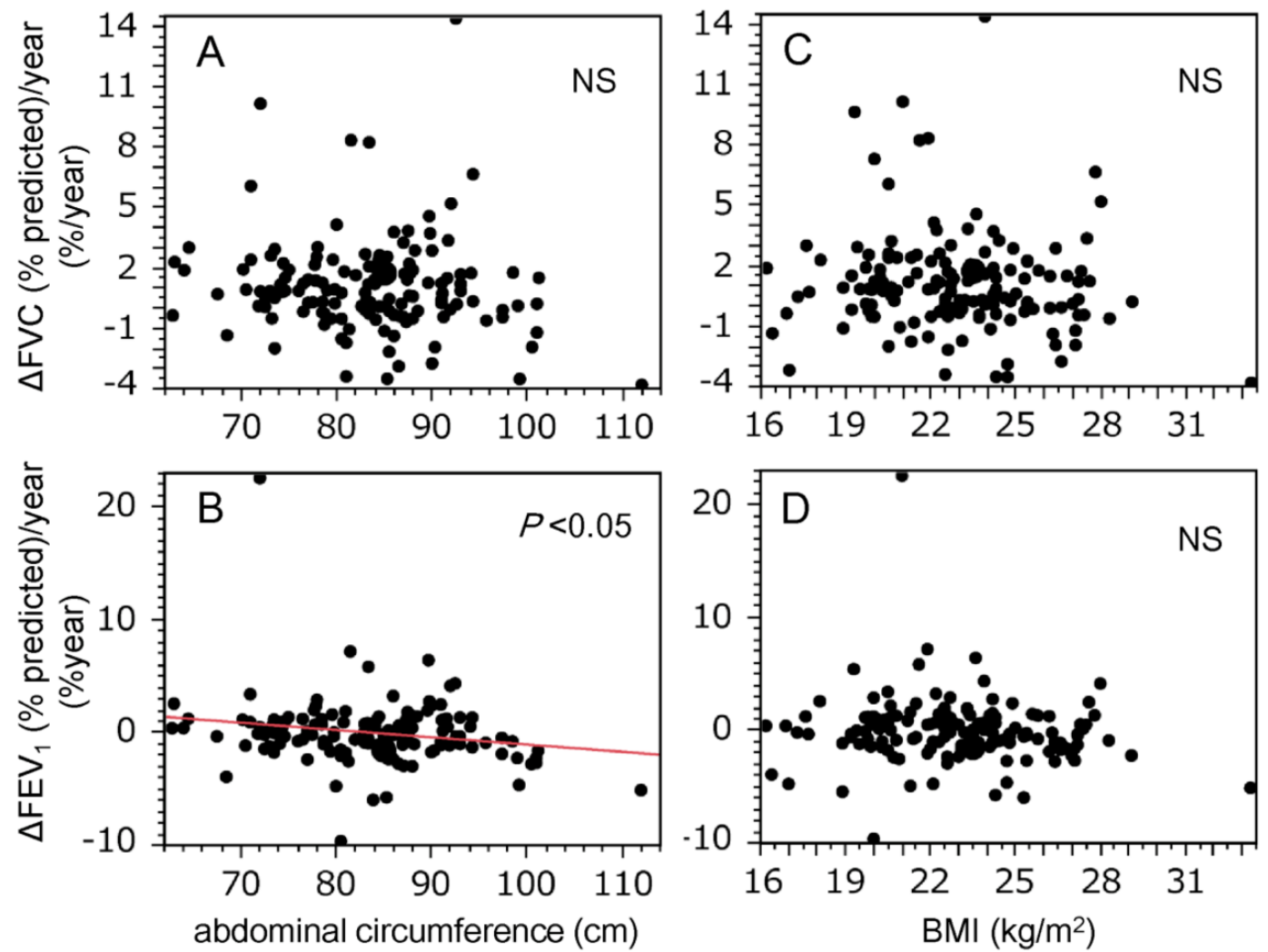

Figure I. Abdominal circumference was inversely correlated with decline in FEV $\mathbf{I}$ in this study population. Graphs show the relationships between decline in spirometric parameters $[\mathrm{A}$ and $\mathrm{C}, \Delta \mathrm{FVC}$ (\% predicted)/year; $\mathrm{B}$ and $\mathrm{D}, \Delta \mathrm{FEV}$ ( $\%$ predicted)/year] and markers of body size ( $A$ and $B$, abdominal circumference; $C$ and $D, B M I)$. Correlations between spirometric measurements and markers of body size were evaluated using Pearson's product moment correlation coefficient. A statistically significant relationship was only observed for $\triangle \mathrm{FEV}$ ( \% predicted)/year and abdominal circumference $(\mathrm{B}, r=-0.19, P=0.024)$. BMI, body mass index; FVC, forced vital capacity; FEV, forced expiratory volume in I s. 
Table 4. Differences in subject characteristics according to presence or absence of metabolic syndrome (Mets).

\begin{tabular}{lll}
\hline & Mets- $(\mathrm{n}=120)$ & Mets+ $(\mathrm{n}=17)$ \\
\hline Age, years & $60.2(8.0)$ & $56.5(8.2)^{*}$ \\
Brinkman index, cigarettexyear & $827.9(408.9)$ & $955.8(733.5)$ \\
BMI, kg/m² & $22.4(2.5)$ & $26.9(2.0)^{* * *}$ \\
$\Delta$ BMI, kg/m² & $-0.01(0.10)$ & $0.89(0.27)^{* *}$ \\
AC, cm & $81.6(7.4)$ & $95.9(5.7)^{* * *}$ \\
Systolic BP, mm Hg & $132.6(19.1)$ & $144.9(16.8)^{*}$ \\
Diastolic BP, mm Hg & $79.9(11.7)$ & $88.1(9.9)^{* *}$ \\
TG, mg/dL & $134.0(121.5)$ & $197.2(72.8)^{*}$ \\
HDL-c, mg/dL & $54.7(13.6)$ & $43.5(11.3)^{*}$ \\
FBS, mg/dL & $96.3(22.6)$ & $100.8(16.7)$
\end{tabular}

Out of 147 subjects, $\mathrm{AC}$ and FBS data were not available for 10 subjects. $\triangle \mathrm{BMI}=\mathrm{BMI}_{\mathrm{visit} 2}-\mathrm{BMI}_{\mathrm{visit1}}$. Values are means (SD). Differences in variables between subjects with Met and those without Mets were evaluated by Student's t-test. ${ }^{*} P<0.05,{ }^{* *} P<0.001,{ }^{* * *} P<0.0001$ compared with Mets-. BMI, body mass index; AC, abdominal circumference; BP, blood pressure; TG, triglyceride; HDL-c, high-density lipoprotein cholesterol; FBS, fasting blood sugar.

Table 5. Prevalence of decline in FEV (\% predicted) according to the presence or absence of metabolic syndrome (Mets).

\begin{tabular}{lll}
\hline FEV 1 & Mets $-(\mathrm{n}=120)$ & Mets $+(\mathrm{n}=17)$ \\
\hline Decline, $\mathrm{n}(\%)$ & $18(15)$ & $6(35.3)$ \\
No decline, $\mathrm{n}(\%)$ & $102(85)$ & $11(64.7)$ \\
\hline
\end{tabular}

$P=0.039$, chi-square test. $\mathrm{FEV}_{1}$, forced expiratory volume in $1 \mathrm{~s}$.

Table 6. Decline in FEV and spirometric measures at visit 2 according to the presence or absence of metabolic syndrome (Mets).

\begin{tabular}{llll}
\hline & Mets $-(\mathrm{n}=120)$ & Mets $+(\mathrm{n}=17)$ & P value \\
\hline$\Delta \mathrm{FEV}_{1}$ (\% predicted)/year, \%/year & $0.04(-1.02,1.14)$ & $-1.11(-2.21,0.07)$ & 0.04 \\
$\mathrm{FVC}_{1} \%$ predicted & $100.1(13.3)$ & $94.1(15.5)$ & 0.09 \\
$\mathrm{FEV}_{1}, \%$ predicted & $93.6(16.3)$ & $88.0(12.2)$ & 0.17 \\
$\mathrm{FEV}_{1} / \mathrm{FVC}, \%$ & $73.3(9.5)$ & $74.3(6.2)$ & 0.68 \\
\hline
\end{tabular}

Values are median (interquartile range) or mean (SD). Difference in $\triangle \mathrm{FEV}_{1}$ (\% predicted)/year between subjects with Mets and those without Mets was evaluated by the Mann-Whitney U test. Differences in other variables were evaluated by Student's t-test. FVC, forced vital capacity; FEV $_{1}$, forced expiratory volume in $1 \mathrm{~s}$

\section{Discussion}

In this longitudinal study, spirometric values were measured twice in male smokers, who participated in an annual health check. At visit 2, AC was evaluated in these subjects, and a significant correlation between rate of decline in $\mathrm{FEV}_{1}$ and $\mathrm{AC}$ was retrospectively demonstrated. Furthermore, multivariate logistic regression analysis revealed that a decline in $\mathrm{FEV}_{1}$ was associated with $\mathrm{AC}$, independently of age, cigarette consumption (Brinkman index) and $\triangle \mathrm{BMI}$. In addition, a decline in $\mathrm{FEV}_{1}$ was more prevalent among subjects with Mets, and the rate of decline in $\mathrm{FEV}_{1}$ in these subjects was significantly greater than that in subjects without Mets.

Mets is a common disease that is characterized by abdominal obesity, high blood pressure, hypertriglyceridemia, low HDL-c levels, and high fasting glucose levels (24). Data from the Third National
Health and Nutrition Examination Survey indicates that this syndrome is present in almost a quarter of US residents aged 20 years or older (25). Although Mets is less prevalent in Asian compared with Western populations, a survey of the incidence of acute myocardial infarction suggested that the prevalence of Mets is also increasing in Japan, resulting in an increased incidence of myocardial infarction among men (26). Mets is associated with an increased risk of diabetes mellitus and cardiovascular disease, leading to increased cardiovascular and all-cause mortality (14). Recent studies have demonstrated that systemic inflammation, derived from visceral fat, is an important mechanism in the pathogenesis of Mets (13). Because both Mets and COPD are associated with the same comorbidities, including atherosclerosis and diabetes, and involve a state of chronic systemic inflammation, the pathogenesis of the two diseases is thought to share a common inflammatory pathway. A recent 
study showed that the prevalence of Mets is greater in subjects with COPD compared to those without COPD $(11,27)$.

The mechanism underlying the rapid decline in pulmonary function in male smokers with a high AC has not been fully elucidated. One possible mechanism that might explain this phenomenon is endothelial dysfunction in subjects with Mets (28). Accumulating evidence suggests that endothelial dysfunction is one of the important features in the development of COPD (29). In particular, pulmonary endothelial cell apoptosis is thought to play an important role in the loss of pulmonary vasculature $(30,31)$. Systemic inflammation may play a significant role in endothelial dysfunction in COPD patients (32). Mets is characterized by systemic low-grade inflammation, and endothelial dysfunction resulting from this systemic inflammation leads to functional impairment of organ systems $(33,34)$. In addition, long term exposure to cigarette smoke induces systemic inflammation (35). Therefore, in active smokers with Mets, systemic inflammation may be enhanced because it is derived from two sources, namely visceral fat and exposure of the lungs to cigarette smoke. This may result in enhanced endothelial dysfunction and a rapid decline in pulmonary function.

Abdominal obesity may change the compliance of the diaphragm and chest wall. Chen and colleagues reported that a $1-\mathrm{cm}$ increase in $\mathrm{AC}$ was associated with a $13-\mathrm{mL}$ reduction in $\mathrm{FVC}$ and a $11-\mathrm{mL}$ reduction in $\mathrm{FEV}_{1}$ (23). In the present study, there was no difference in mean BMI between visit 1 and visit 2, although $\mathrm{AC}$ was not measured at visit 1 . In addition, multivariate logistic regression analysis demonstrated that $A C$ was a significant factor for discrimination of decline in $\mathrm{FEV}_{1}$, independently of $\triangle \mathrm{BMI}$ (Table 3). It is unlikely that the decline in $\mathrm{FEV}_{1}$ observed in the present study is explained by weight gain during follow-up.

In the present study, the longitudinal decrease in $\mathrm{FEV}_{1}$ was significantly greater in subjects with Mets, and the prevalence of a decline in $\mathrm{FEV}_{1}$ was significantly greater in subjects with Mets than in those without Mets. However, $\mathrm{FEV}_{1} / \mathrm{FVC}$ did not differ significantly between subjects with or without Mets. Even after adjustment of $\mathrm{FEV}_{1} / \mathrm{FVC}$ for age, there was no difference in $\mathrm{FEV}_{1} / \mathrm{FVC}$ between the two groups. This finding was in keeping with those from previous studies, which demonstrated that $\mathrm{FEV}_{1} / \mathrm{FVC}$ was not reduced in subjects with Mets compared to those without Mets $(36,37)$.

This study had several limitations. First, there may have been sampling bias. Takahata town has a resident population of 15,222 adults aged 40 years or older. For visit 1, data for a total of 3,257 enrolled subjects was entered into the final statistical analysis. Among the 1,502 male subjects, there were 523 active smokers. However, follow-up spirometry in 2009 was only performed for 147 subjects who had continued to smoke; the remaining subjects were lost to follow-up or quit smoking before visit 2 . The number of subjects who were followed-up was not large, but the only significant difference between participants and non-participants in 2009 was age (see Method section). Thus, we thought the participants in 2009 were not largely different from non-participants. Differences between participants and non-participants in type of employment, socio-economic status or life style may have influenced the results. However, this information was not available in the present study. Second, pulmonary function testing at visit 2 in 2009 was only performed for male current smokers, not for male non-smokers, ex-smokers or females. This was only because we could not find a sufficient number of testers in 2009. Therefore we needed to focus on the data for the subjects who were assessed in the present study. Because of this limitation, the results from the present study may not be applicable to females or ex-smokers. Third, AC measurements were performed at visit 2 , not visit 1 . Therefore, the association between decline in $\mathrm{FEV}_{1}$ and $\mathrm{AC}$ was retrospectively demonstrated in this study. It should be noted that it could not be concluded that $\mathrm{AC}$ is predictive for decline in $\mathrm{FEV}_{1}$ among male smokers. However, despite these limitations we think this study is clinically significant as an initial investigation, and future prospective studies have the potential to confirm AC as a new and simple biomarker for discriminating subjects with a decline in $\mathrm{FEV}_{1}$ among active smokers.

In conclusion, $\mathrm{AC}$ was significantly associated with the rate of decline in $\mathrm{FEV}_{1}$ among male smokers in this study population. A decline in $\mathrm{FEV}_{1}$ was more prevalent among subjects with Mets, and the rate of decline in $\mathrm{FEV}_{1}$ was significantly greater in subjects with Mets compared to those without Mets. This retrospective analysis suggested that measurement of AC may be a simple and useful method of discriminating subjects with a decline in $\mathrm{FEV}_{1}$ among male smokers.

\section{Acknowledgments}

We thank Taiko Aita, Emiko Nakamura, and Eiji Tsuchida for their excellent technical assistance. We also thank the additional contributors: Michiko Nishiwaki (Yamagata City Hospital, Saiseikan), Toshihiro Wada (Yamagata City Hospital, Saiseikan), Jun-Ichi Machiya (Nihonkai General Hospital), Noriyuki Hirama (NHO Yamagata National Hospi- 
tal), Noriaki Takabatake (Tohoku Central Hospital), Makoto Sata (National Cerebral and Cardiovascular Center, Japan).

\section{Funding}

This study was supported by a grant-in-aid from the Global COE Program of the Japan Society for the Promotion of Science, and grants-in-aid for Scientific Research from the Ministry of Education, Culture, Sports, Science and Technology, Japan (19590880, 20590892, and 23390220).

\section{Ethics approval}

This study was approved by the institutional ethics committee and all participants gave written informed consent.

\section{Conflicts of interest}

The authors have no conflicts of interest to disclose.

\section{References}

1 [Internet] Global initiative for chronic obstructive lung disease. Global strategy for the diagnosis, management and prevention of chronic obstructive pulmonary disease. http://www.goldcopd.org/.

2 Iwamoto H, Yokoyama A, Kitahara Y, et al. Airflow limitation in smokers is associated with subclinical atherosclerosis. Am J Respir Crit Care Med. 2009; 179: 35-40.

3 Maclay JD, McAllister DA, Macnee W. Cardiovascular risk in chronic obstructive pulmonary disease. Respirology. 2007; 12: 634-41.

4 Maggi S, Siviero P, Gonnelli S, et al. Osteoporosis risk in patients with chronic obstructive pulmonary disease: The eolo study. J Clin Densitom. 2009; 12: 345-52.

5 Mannino DM, Thorn D, Swensen A, et al. Prevalence and outcomes of diabetes, hypertension and cardiovascular disease in COPD. Eur Respir J. 2008; 32: 962-9.

6 Takabatake N, Sata M, Inoue S, et al. A novel polymorphism in secretory phospholipase A2-IID is associated with body weight loss in chronic obstructive pulmonary disease. Am J Respir Crit Care Med. 2005; 172: 1097-104.

7 Wouters EF, Creutzberg EC, Schols AM. Systemic effects in COPD. Chest. 2002; 121: 127S-30S.

8 Yao H, Rahman I. Current concepts on the role of inflammation in COPD and lung cancer. Curr Opin Pharmacol. 2009; 9: 375-83.

9 Clini E, Crisafulli E, Radaeli A, et al. COPD and the metabolic syndrome: An intriguing association. Intern Emerg Med. 2011; Epub ahead of print.

10 Lam KB, Jordan RE, Jiang CQ, et al. Airflow obstruction and metabolic syndrome: The Guangzhou Biobank Cohort Study. Eur Respir J. 2010; 35: 317-23.

11 Marquis K, Maltais F, Duguay V, et al. The metabolic syndrome in patients with chronic obstructive pulmonary disease. J Cardiopulm Rehabil. 2005; 25: 226-32.

12 Watz H, Waschki B, Kirsten A, et al. The metabolic syndrome in patients with chronic bronchitis and COPD: Frequency and associated consequences for systemic inflammation and physical inactivity. Chest. 2009; 136: 1039-46.

13 Calabro P, Yeh ET. Intra-abdominal adiposity, inflammation, and cardiovascular risk: New insight into global cardiometabolic risk. Curr Hypertens Rep. 2008; 10: 32-8.

14 Mottillo S, Filion KB, Genest J, et al. The metabolic syndrome and cardiovascular risk a systematic review and meta-analysis. J Am Coll Cardiol. 2010; 56: 1113-32.

15 Leone N, Ducimetiere P, Gariepy J, et al. Distension of the carotid artery and risk of coronary events: The Three-City study. Arterioscler Thromb Vasc Biol. 2008; 28: 1392-7.

16 Osaka D, Shibata Y, Abe S, et al. Relationship between habit of cigarette smoking and airflow limitation in healthy Japanese individuals: The Takahata study. Intern Med. 2010; 49: 1489-99.
17 Aida Y, Shibata Y, Osaka D, et al. The relationship between serum uric acid and spirometric values in participants in a health check: The Takahata study. Int J Med Sci. 2011; 8: 470-8.

18 Kishi H, Shibata Y, Osaka D, et al. FEV6 and FEV1/FEV6 in Japanese participants of the community-based annual health check: The Takahata study. Intern Med. 2011; 50: 87-93.

19 Nemoto T, Shibata Y, Osaka D, et al. Impact of cigarette smoking on maximal expiratory flows in a general population: The Takahata study. Intern Med. 2011; 50: 2547-55.

20 Shibata Y, Watanabe T, Osaka D, et al. Impairment of pulmonary function is an independent risk factor for atrial fibrillation: The Takahata study. Int J Med Sci. 2011; 8: 514-22.

21 Ninomiya T, Kiyohara Y, Kubo M, et al. Metabolic syndrome and CKD in a general Japanese population: The Hisayama study. Am J Kidney Dis. 2006; 48: 383-91.

22 The Committee of Pulmonary Physiology JRS. Guidelines for pulmonary function tests: Spirometry, flow-volume curve, diffusion capacity of the lung. Tokyo, 2004.

23 Chen Y, Rennie D, Cormier YF, et al. Waist circumference is associated with pulmonary function in normal-weight, overweight, and obese subjects. Am J Clin Nutr. 2007; 85: 35-9.

24 Expert Panel on Detection E and Treatment of High Blood Cholesterol in Adults. Executive summary of the third report of the national cholesterol education program (NCEP) expert panel on detection, evaluation, and treatment of high blood cholesterol in adults (adult treatment panel III). JAMA. 2001; 285: 2486-97.

25 Ford ES, Giles WH, Dietz WH Prevalence of the metabolic syndrome among US adults: Findings from the third national health and nutrition examination survey. JAMA. 2002; 287: 356-9.

26 Nishiyama S, Watanabe T, Arimoto T, et al. Trends in coronary risk factors among patients with acute myocardial infarction over the last decade: The Yamagata AMI Registry. J Atheroscler Thromb. 2010; 17: 989-98.

27 Park BH, Park MS, Chang J, et al. Chronic obstructive pulmonary disease and metabolic syndrome: A nationwide survey in Korea. Int J Tuberc Lung Dis. 2012; 16: 694-700.

28 Devaraj S, Jialal I. Dysfunctional endothelial progenitor cells in metabolic syndrome. Exp Diabetes Res. 2012; 2012: 585018.

29 Arao T, Takabatake N, Sata M, et al. In vivo evidence of endothelial injury in chronic obstructive pulmonary disease by lung scintigraphic assessment of (123)I-metaiodobenzylguanidine. J Nucl Med. 2003; 44: 1747-54.

30 Kasahara Y, Tuder RM, Cool CD, et al. Endothelial cell death and decreased expression of vascular endothelial growth factor and vascular endothelial growth factor receptor 2 in emphysema. Am J Respir Crit Care Med. 2001; 163: 737-44.

31 Kasahara Y, Tuder RM, Taraseviciene-Stewart L, et al. Inhibition of VEGF receptors causes lung cell apoptosis and emphysema. J Clin Invest. 2000; 106: 1311-9.

32 Eickhoff P, Valipour A, Kiss D, et al. Determinants of systemic vascular function in patients with stable chronic obstructive pulmonary disease. Am J Respir Crit Care Med. 2008; 178: 1211-8.

33 Takeno M, Yasuda S, Otsuka Y, et al. Impact of metabolic syndrome on the long-term survival of patients with acute myocardial infarction: Potential association with C-reactive protein. Circ J. 2008; 72: 415-9.

34 Thompson AM, Zhang $\mathrm{Y}$, Tong $\mathrm{W}$, et al. Association of inflammation and endothelial dysfunction with metabolic syndrome, prediabetes and diabetes in adults from inner mongolia, china. BMC Endocr Disord. 2011; 11: 16.

35 Tracy RP, Psaty BM, Macy E, et al. Lifetime smoking exposure affects the association of c-reactive protein with cardiovascular disease risk factors and subclinical disease in healthy elderly subjects. Arteriosclerosis Thrombosis and Vascular Biology. 1997; 17: 2167-76.

36 Nakajima K, Kubouchi Y, Muneyuki T, et al. A possible association between suspected restrictive pattern as assessed by ordinary pulmonary function test and the metabolic syndrome. Chest. 2008; 134: 712-8.

37 Yeh F, Dixon AE, Marion S, et al. Obesity in adults is associated with reduced lung function in metabolic syndrome and diabetes: The Strong Heart Study. Diabetes Care. 2011; 34: 2306-13. 